\title{
Evaluation of Dynamic Stress-Strain Relations of Ti-Alloys and Al-Alloys Based on the Thermally Activated Process Concept*
}

\author{
Kinya OGAWA**
}

\begin{abstract}
Since titanium and aluminum alloys are the most promising structural materials for the high velocity vehicles, the impact tensile strength of the materials is presently investigated. Three kinds of aging treatments on the beta-titanium alloy and two on the 6061 aluminum alloy were performed, and the tensile deformation behaviors were identified in the wide range of the temperature and the strain rate. The stress-strain relations of the titanium alloy significantly depend on the temperature and the strain rate investigated. Thermally activated process concept was applied to explain the experimental results, and the stress-strain relations at high strain rates were well understood with taking account of adiabatic heating effect. In the case of the aluminum alloy, the temperature and the strain rate effects are significant only in the low temperature range. Both for the alloys investigated, the stress-strain curves depend on the microstructures, while the temperature and the strain rate effects are almost independent of the different aging treatments.
\end{abstract}

Key Words: Constitutive Equation, Impact Strength, Tensile Properties, Heat Treatments, Split Hopkinson Bar, Titanium Alloy, Aluminum Alloy, Thermal Activation, Temperature Strain Rate Parameter

\section{Introduction}

Titanium and aluminum alloys are widely used for aircrafts because of its high strength-weight ratio and excellent corrosion resistance, and will be applied to ground vehicles for saving energy demands. Therefore, the dynamic deformation characterization of these materials is increasingly needed from the impact crashworthiness view point. At low strain rates it is usually confirmed that the thermally activated process controls plastic deformation, but it is still not evident how far it can be extended. It is not always reasonable to apply the viscous drag of high velocity dislocations in explanation of significant increase of strength at high strain rates. Clifton ${ }^{(1)}$ concluded that the viscous drag could not be expected at strain rates less than $10^{4} / \mathrm{s}$ and Armstrong and Zerilli ${ }^{(2)}$ successfully correlated their Taylor test results on Armco iron with thermally activated flow. Follansbee ${ }^{(3)}$ pointed

* Received 18th November, 2002 (No.02-4249)

** Division of Aeronautics and Astronautics, Graduate School of Engineering, Kyoto University, Yoshida Honmachi, Sakyo-ku, Kyoto 606-8501, Japan. E-mail : ogawa@kuaero.kyoto-u.ac.jp out that temperature and strain rate history effect often veiled deformation behaviors of soft materials controlled by thermal activation. Holt et al. ${ }^{(4)}$ suggested that the strengthening effect of alloying is almost entirely due to an increase in the athermal stress component and the rate sensitivity would be relatively smaller for a strong alloy. The present author has investigated the temperature and strain rate effect on the stress ${ }^{-}$strain relations of several titanium alloys and has clarified the thermally activated process concept to be applied for deformation behavior up to the strain rate of $10^{4} / \mathrm{s}^{(5),(6)}$. In the case of aluminum alloy, the relatively insignificant strain rate effect has been reported in the range of strain rate up to $10^{3} / \mathrm{s}$, while the upturn of the effect was found beyond at the strain rate $^{(7)}$.

In the present paper, temperature and strain rate effects on the tensile stress-strain relations of titanium and aluminum alloys were investigated in the wide ranges of temperature from $77 \mathrm{~K}$ to $573 \mathrm{~K}$ and of strain rates from $10^{-4} / \mathrm{s}$ to $10^{3} / \mathrm{s}$. The deformation behaviors will be discussed with respect to the effect of heat treatment using the thermally activated process concept. 


\section{Experimentation}

The materials used are 15-3-3-3 beta-titanium alloy and $A 6061$ aluminum alloy. Three kinds of heat treatments were performed for titanium alloy after the solid solution treatment as; (A) aged for 6 hours at $723 \mathrm{~K}$, (B) aged for 8 hours at $813 \mathrm{~K}$, and (C) aged for 72 hours at $573 \mathrm{~K}$ followed by 4 hours aging at 813 $\mathrm{K}$. The aluminum alloy was water quenched after solution treatment at $793 \mathrm{~K}$ and was 8 hour-tempered at $448 \mathrm{~K}$ for $\mathrm{T} 6$-treatment, and was subsequently annealed at $693 \mathrm{~K}$ for 2 hours followed by furnace cooling for $\mathrm{O}$-treatment. The specimen was machined from the material and the tensile axis was in the extruded direction. The strain was evaluated using the gage length of $5 \mathrm{~mm}$ as the parallel portion of the specimen.

Quasi-static test in the strain rate of $10^{-4}-10^{-2} / \mathrm{s}$ was performed using the universal testing machine, and the strain rate change test to evaluate the strain rate sensitivity of flow stress was carried out as well as the constant strain rate test. Split Hopkinson pressure bar apparatus shown in Fig. 1 was used to perform the impact tensile test at the strain rate above $10^{2} / \mathrm{s}$. The specimen depicted in Fig. 1 was screwed to the loading bars. The stress waves in the input and the output bars were sensed by strain gauges cemented on the indicated positions in the figure, and were stored in the multi-channels transient recorder with sampling rate of 1 word/ $\mu$ s after amplified up to $60 \mathrm{~dB}$ through the pre-amplifier. The stress, strain, and strain rate were analyzed in digital form by using the one-dimensional stress wave theory as usual. A cooling vessel and an electric furnace were used for low and high temperature tests, respectively. Alumel-chromel thermocouples were attached to specimens to sense the temperature controlled with
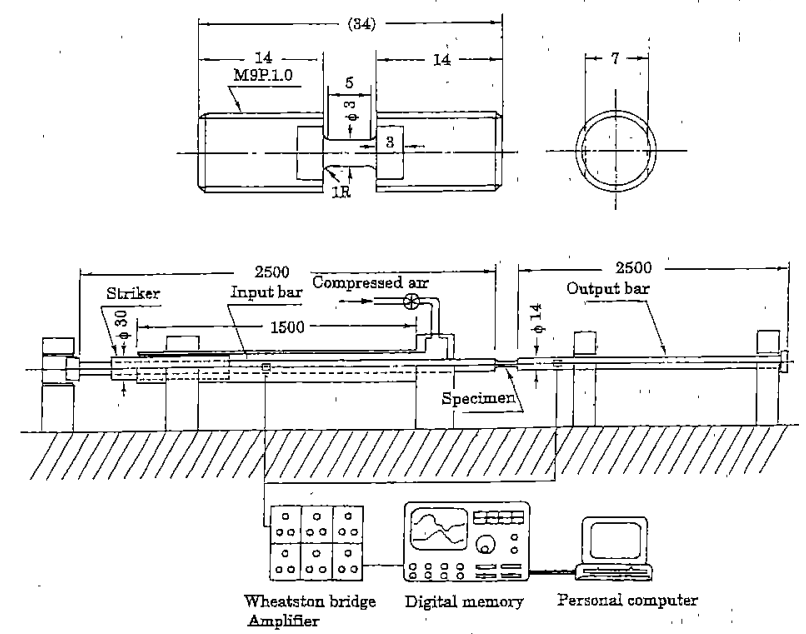

Fig. 1 Experimental set-up for dynamic test and shape of specimen in $\pm 1 \mathrm{~K}$. In the case of low strain rates, loading attachments and a specimen were kept at the respective test temperatures for 15 minutes to depress fluctuations of temperature, before the test run.

\section{Experimental Results}

Nominal stress ${ }^{-}$strain relations obtained at static and dynamic strain rates are represented in Figs. 2 and 3 for titanium alloys and aluminum alloys, respectively. In titanium alloys, stress is increased with lowering temperatures and with increase of strain rate. Work-hardening rate is almost independent of temperature at low strain rate, while significant work-softening is observed at low temperatures in dynamic deformation. For highly hardened material (A), brittle fracture occurs at very small strains at low temperatures. In aluminum alloys, on the other hand, work-hardening rate depends on the temperature and strain rate, but there was not observed any work-softening in uniformly straining region up to the maximum stress even for the dynamic test at low temperatures. An enlargement in the figure indicates
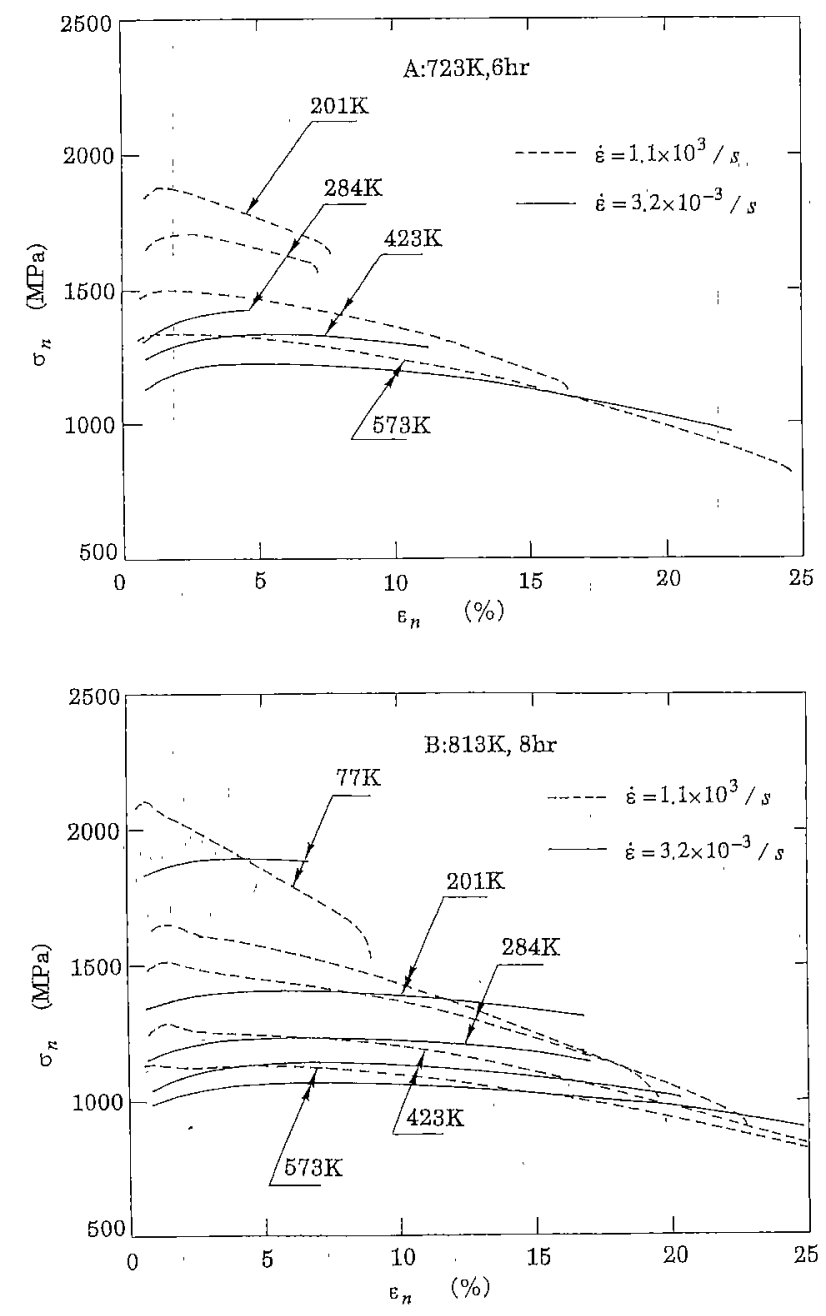

Fig. 2 Stress-strain curves of beta-titanium alloys 
abrupt stress response in the strain rate change during static strain rate deformation. When the strain rate is increased, a slight increase was associated, but it is quickly diminished and a stress-strain relation returns to that at the strain rate $\dot{\varepsilon}_{1}$. The flow stress at around the room temperature is interpreted almost independent of the strain rate as reported by many authors. In the case of the annealed material, since serration phenomena due to the Portevin-Le Chatelie effect was observed in the quasi-static test at temperatures from $289 \mathrm{~K}$ to $373 \mathrm{~K}$, the stress-strain curves were drawn smoothly connecting the mean values of stress amplitude. The strain rate sensitivity of the stress at these temperatures was negative, while it is always positive at other temperatures. There was not any serrated stress-strain relation at high strain rates independent of temperature.

Figures 4 (a) and (b) represent the relation between the flow stress at 5\% strain and the strain
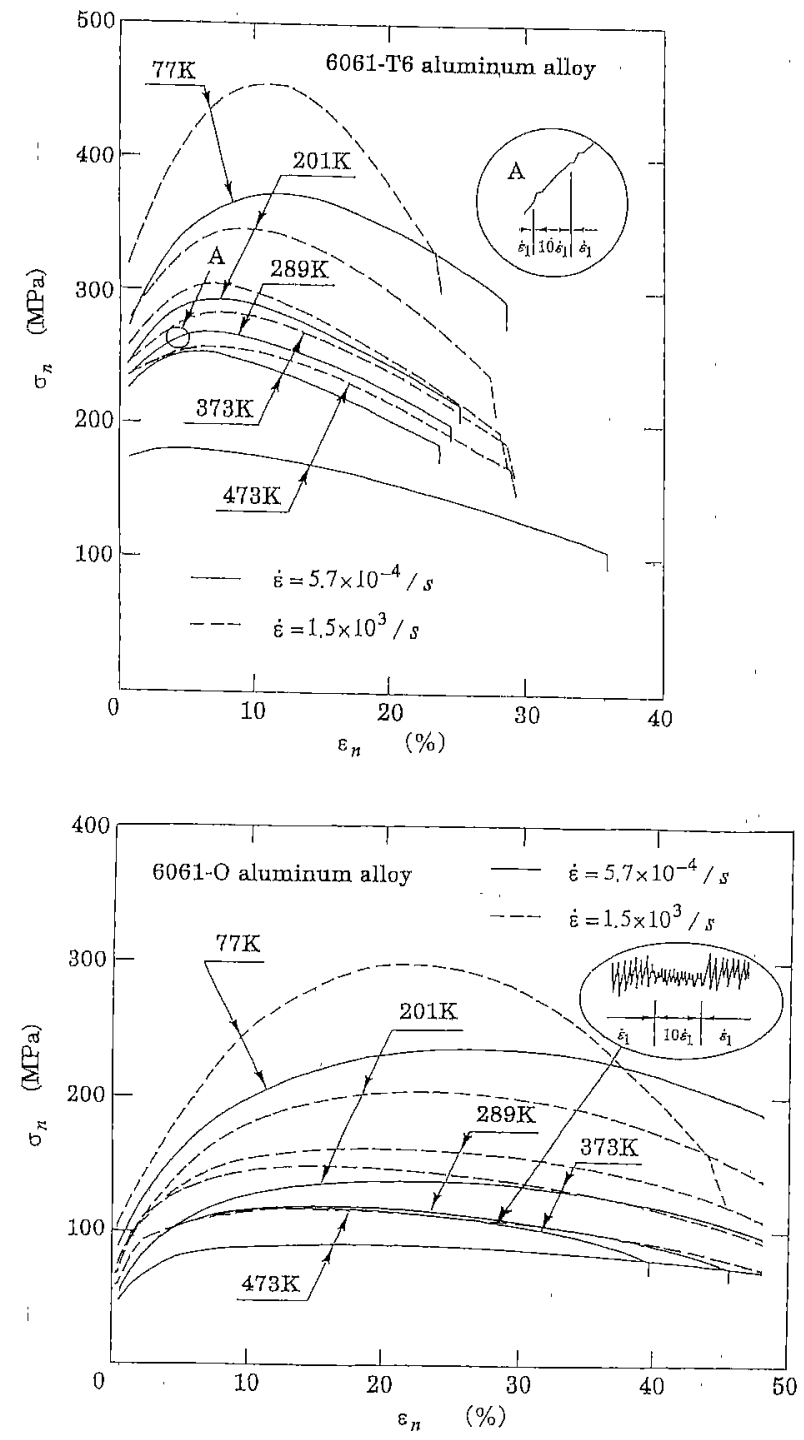

Fig. 3 Stress-strain curves of 6061 aluminum alloy rate for the respective temperatures. The flow stress of titanium alloy increases with a rise of strain rate, and the strain rate sensitivity of stress also increases with a rise of temperature. On the other hand, the flow stress of aluminum alloy increases with an increase of strain rate only at low temperatures. At $289 \mathrm{~K}$, the stress shows almost constant in the low strain rate range, but it tends to increase in high strain rate range of $10^{3} / \mathrm{s}$. Solid lines in the figures are drawn according to the thermally activated process concept and will be explained afterward.

\section{Consideration}

\section{1 Thermally activated process}

The stress, $\sigma$, can be expressed in terms of the thermal, $\sigma^{*}$, and the athermal component, $\sigma_{\mu}$, of the stress as follows.

$$
\sigma=\sigma^{*}+\sigma_{\mu}
$$

The strain rate, $\dot{\varepsilon}$, of a single thermally activated flow can be expressed as ${ }^{(8)}$

$$
\begin{aligned}
\dot{\varepsilon} & =b \rho_{m} L v_{0} \exp \left\{-\left(H_{0}-\int v^{*} d \sigma^{*}\right) / k T\right\} \\
& =\dot{\varepsilon}_{0} \exp \left\{-H\left(\sigma^{*}\right) / k T\right\}
\end{aligned}
$$

where $b$, Burger's vector, $\rho_{m}$, mobile dislocation density, $L$, average distance of short range obstacles, $v_{0}$, part of Debye-frequency, $H_{0}$, the total activation energy, $v^{*}$, the activation volume, $k$, Boltzman constant, and $T$ is the absolute temperature, respectively. Since the activation energy, $H$, is a function of $\sigma^{*}$, the stress, $\sigma$, is rewritten in terms of the temperaturestrain rate parameter, $\xi$, as ;

where

$$
\sigma=\sigma_{\mu}+H^{-1}\left(\sigma^{*}\right)=\sigma(\xi, \varepsilon)
$$

$$
\xi=k T\left(\ln \dot{\varepsilon}_{0}-\ln \dot{\varepsilon}\right)
$$

Figure 5 shows the stress at 5\% strain to temperature relations for three kinds of titanium alloys vertically shifting the relations to adjust the stress values obtained at $573 \mathrm{~K}$. Respective single smooth curves can be drawn for the quasi static and the dynamic results. This fact suggests that even though the athermal component of stress is different, the thermal component of stress is almost identical for the respective heat treatments. Consequently, temperature and strain rate effect on the stress-strain relations can be uniquely determined independent of heat treatment.

Kocks et al. ${ }^{(9)}$ have proposed a general form of the activation energy in terms of the thermal component of stress as follows;

$$
H=H_{0}\left[1-\left(\sigma^{*} / \sigma_{0}^{*}\right)^{p}\right]^{q}, \quad H_{0}=k \xi_{0}
$$

where $0 \leq p \leq 1,1 \leq q \leq 2, \sigma_{0}^{*}$ is the value of $\sigma^{*}$ at $0 \mathrm{~K}$, and $\xi_{0}$ is the value of $\xi$ at $\sigma^{*}=0$. The thermal component of stress in compressive deformation of beta-titanium alloys is almost independent of temperature and strain rate history effect and can be well 

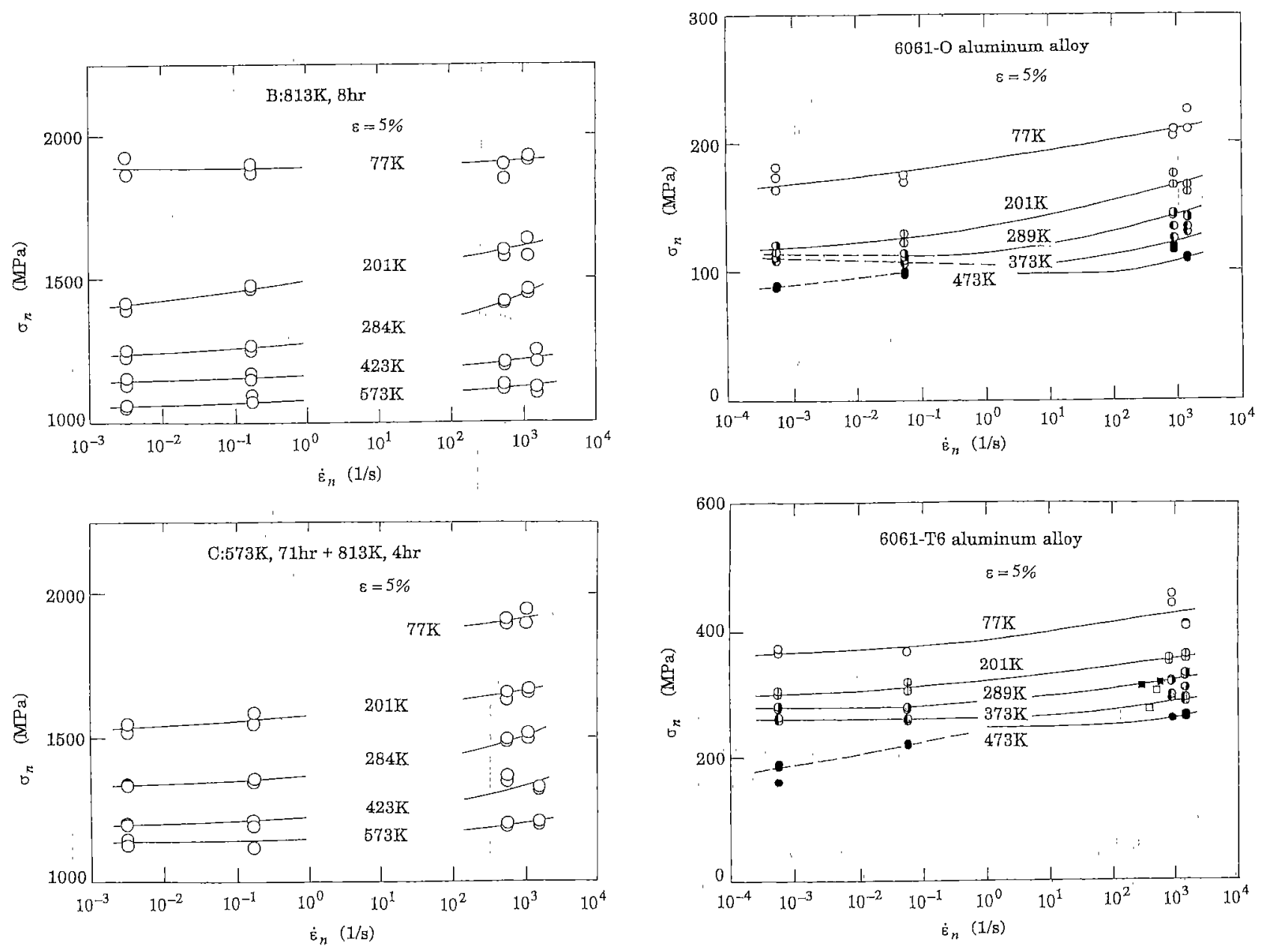

(a)

(b)

Fig. 4 Stress-strain rate relations for titanium alloy (a), and for aluminum alloy (b)

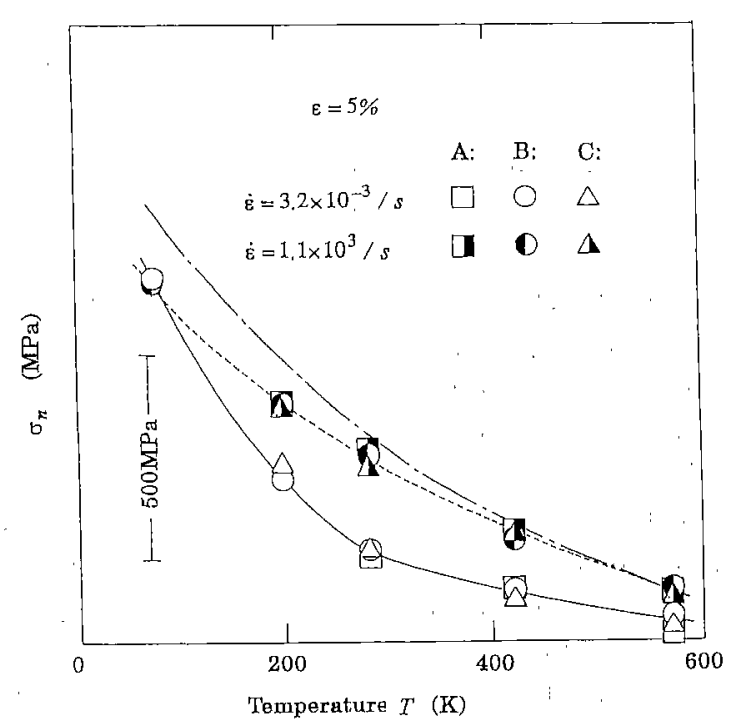

Fig. 5 Temperature and strain rate dependence of stress of titanium alloys at $5 \%$ strain

represented by the form expressed above, while the athermal component of stress can be evaluated as the stress at $\xi_{0}$ and is expressed as;

$$
\sigma_{\mu}=A \varepsilon^{m}
$$

For the present tensile results, the same forms can be reasonably assumed with taking the parameter, $\ln \dot{\varepsilon}_{0}$ $=22$, at low temperatures. Chained curve is predicted for the dynamic test, but it differs to the experiments at low temperatures. This will be understood by adiabatic heating effect during dynamic deformation. Since the temperature dependence of elastic modulus is not available for the present titanium alloy, the stress measured is plotted in the figures without being modified, while in the case of aluminum: alloy, the stress is modified as $\left(E_{T 0} / E_{T}\right) \sigma$, as usual, by taking account of temperature dependence of elastic modulus $^{(10)}$ where $E_{T 0}$ and $E_{T}$ are the values at $0 \mathrm{~K}$ and $T$, respectively.

In Fig. 6 all stresses measured for aluminum alloy are plotted versus the temperature- ${ }^{-}$strain rate parameter, $\xi$, and the experimental data fit smooth solid curves for the respective strains by adopting $\ln \dot{\varepsilon}_{0}=$ 14. These curves predict the stress to the strain rate relations at temperatures and were already shown in Fig. 4(b). Dotted curves will be explained afterward. 


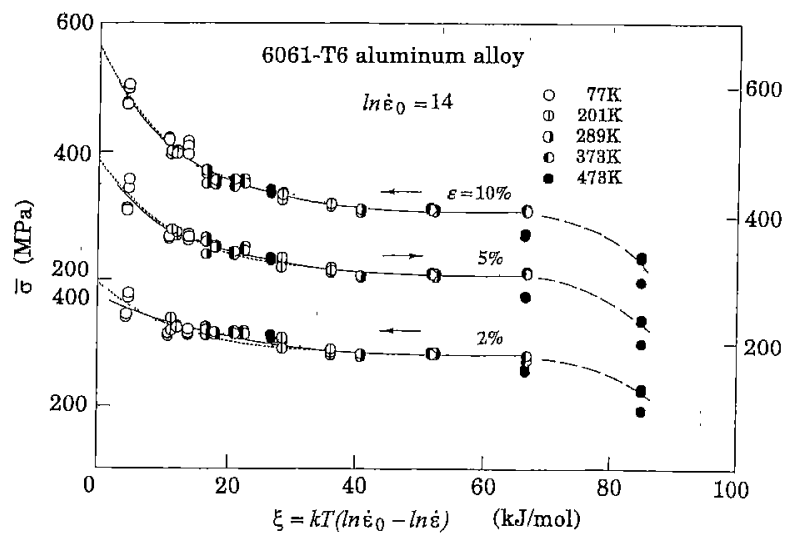

Fig. 6 Stress to temperature-strain rate parameter relation for aluminum alloy

\section{2 Adiabatic heating effect}

Since the temperature dependence of stress is significant in titanium alloys, heat generation during adiabatic deformation at high strain rate will affect deformation behaviors and will yield work softening especially at low temperatures. As was explained above, the thermal component of stress is a function of $\xi$ and the athermal component of stress depends only on the strain, the stress increment, $\Delta \sigma$, can be obtained as ;

$$
\Delta \sigma=\Delta \sigma^{*}+\Delta \sigma_{\mu}=\left(d \sigma^{*} / d \xi\right) \Delta \xi+\left(d \sigma_{\mu} / d \varepsilon\right) \Delta \varepsilon
$$

The temperature rise, $\Delta T$, due to the adiabatic heating can be expressed as ;

$$
\Delta T=\beta \rho \gamma \sigma \Delta \varepsilon
$$

where $0 \leq \beta \leq 1.0, \rho$ is the density of material, $\gamma$ the specific heat. In the split Hopkinson pressure bar method, the strain rate is evaluated in terms of the incident and the transmitted stress waves as ;

$$
\dot{\varepsilon}=2\left(\sigma_{\mathrm{I}}-\left(A_{0} / A_{1}\right) \sigma\right) /\left(\rho_{0} C_{0} l_{0}\right)
$$

with assumption of identical stress state on the both sides of a specimen, where $A_{0}, A_{1}$ are the sectional areas of loading bars and a specimen, $\rho_{0}$ and $C_{0}$ are the density and the elastic wave velocity of the bars, and $l_{0}$ is the initial length of a specimen. When the incident wave is given, the stress, the strain, the strain rate, and the temperature can be evaluated by these equations from $(5)$ to $(9)$. The stress-strain relations of the material (B) for adiabatic condition $(\beta=$ 1.0) are represented by broken curves in Fig. 7 with the experiments (chained curves), and calculated temperature change is typically shown in Fig. 8. It can be seen that the predictions are not so bad, but the work-softening rates obtained in experiments at low temperatures are much less than the predicted. This may be understood by appropriately taking the gage length of the specimen. Since the plastic deformation in the regions adjacent to the fillets of the specimen is constrained to some extent, the effective gage length of about $3 \mathrm{~mm}$. should be adapted from the observa-

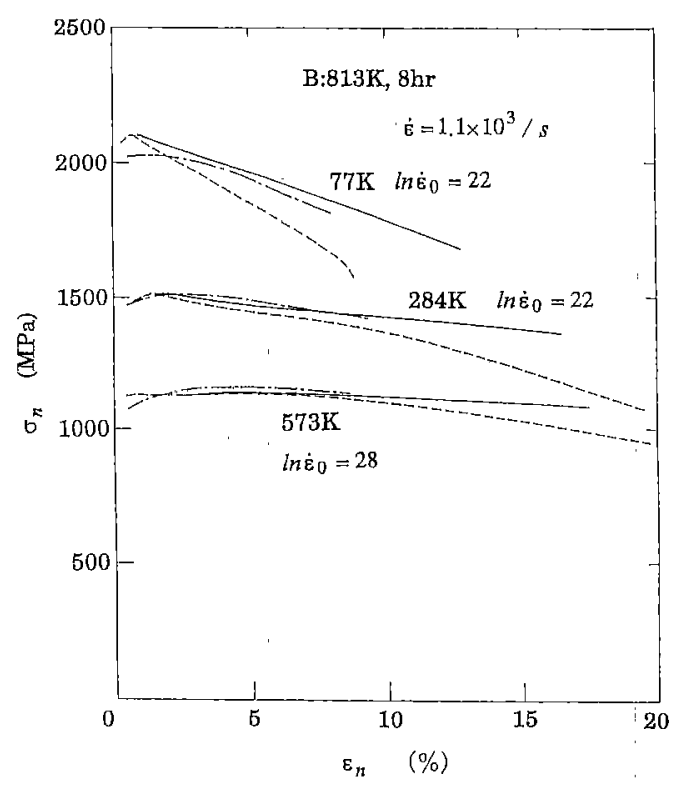

Fig. 7 Adiabatic heating effect on stress-strain curves (calculation and experiment)

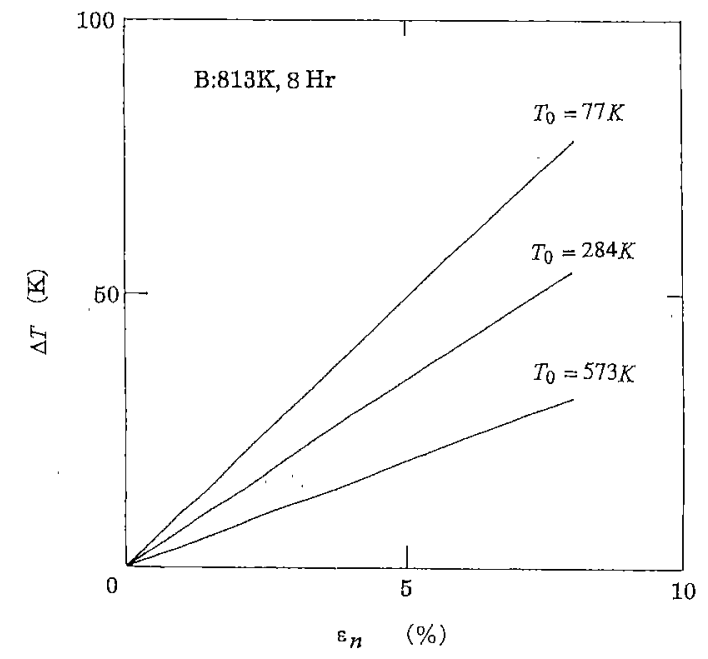

Fig. 8 Adiabatic temperature rise during dynamic deformation (calculation)

tions on the deformed specimen. Solid curves in Fig. 7 represent the predictions based on the effective gage length, and are in fairy good agreement with the experimental results.

\section{3 Dislocation intersection}

Dislocation intersection is most probable deformation mechanism in FCC pure metals such as aluminum, and it may be also applicable for precipitation hardening alloy, since prismatic dislocation loops formed around large precipitates interact with glide dislocations $^{(11)}$. In the case of pure aluminum, activation volume, $v^{*}$, was expressed as follows ${ }^{(12)}$;

$$
v^{*} \sigma^{*}=B
$$

where, $B$ is the experimental constant. The strain rate is now given as; 


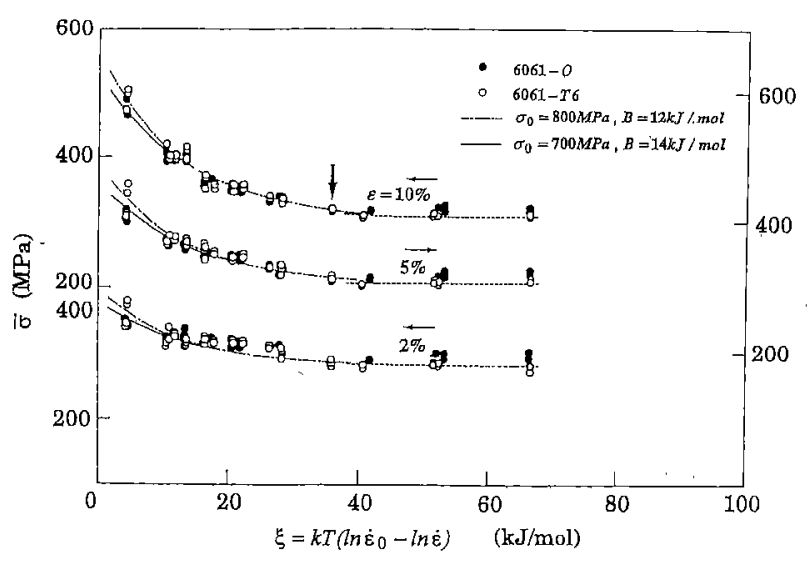

Fig. 9 Effect of heat treatment on the thermal and the athermal component of stress

$$
\begin{aligned}
\dot{\varepsilon} & =b \rho_{m} L v_{0} \exp \left\{-\left(H_{0}-\int_{0}^{\sigma^{*}} v^{*} d \sigma^{*}\right) / k T\right\} \\
& =\dot{\varepsilon}_{0} \exp \left\{-B\left(\ln \sigma_{0}^{*}-\ln \sigma^{*}\right) / k T\right\}
\end{aligned}
$$

It should be noted that the stress, $\sigma_{0}{ }^{*}$, is the thermal component of stress at $T=0$. Then, the thermal component of stress is written as;

$$
\sigma^{*}=\sigma_{0}{ }^{*} \exp (-\xi / B)=\sigma_{0} \varepsilon^{1 / 2} \exp (-\xi / B)
$$

Here, $\sigma_{0}{ }^{*}=\sigma_{0} \varepsilon^{1 / 2}$, since dislocation density is increased with strain ${ }^{(13)}$. Reasonably fitting curves for the respective strains can be drawn as shown by dotted lines in Fig. 6. These curves agree fairly well with the experimental results, and evaluated activation volume of $(1.8 \sim 0.8) \times 10^{-22} \mathrm{~cm}^{3}$, well corresponding to the value for pure aluminum ${ }^{(12)}$. The dislocation intersection is interpreted to control the deformation of the present alloy.

Now, it is relevant to know the effect of heat treatment on the deformation characteristics. Figure 9 shows the stress at the respective strains versus the temperature-strain rate parameter both for the aged and the annealed aluminum alloys. Since the results of aged material were much higher than those of annealed ones, the stress data statically obtained at $201 \mathrm{~K}$ (indicated by an arrow) were shifted to compensate the difference. It is clearly seen that the flow stresses both for the materials are almost identical. This fact implies that the thermal component of stress is almost independent of heat treatment, even though the athermal component of stress is significantly increased in the aged material.

\section{Conclusions}

Tensile stress strain relations of beta-titanium alloy and aluminum alloy are clarified in the temperatures from $77 \mathrm{~K}$ to $573 \mathrm{~K}$ and the strain rates from
$10^{-4} / \mathrm{s}$ to $1.5 \times 10^{3} / \mathrm{s}$, and the following conclusion is obtained.

(1) The stress-strain relations depend on the temperature and the strain rate and are well understood by the thermally activated process concept.

(2) The athermal component of stress is significantly affected by heat treatment, while the thermal component of stress is almost independent of heat treatment and is uniquely characterized by deformation mechanism.

\section{References}

(1) Clifton, R.J., Dynamic Plasticity, J. Appl. Mech., Vol. 50 (1983), pp. 941-952.

(2) Armstrong, R.W. and Zerilli, F.J., Dislocation Mechanics Based Analysis of Material Dynamics Behavior, Proc. DYMAT88., Jour. de Physique, Vol. 49 (1988), C3, pp. 529-534.

(3) Follansbee, P.S., Analysis of the Strain-rate Sensitivity at High Strain Rates in fcc and bcc Metals, Inst. Phys. Conf. Ser., No. 102 (1989), pp. 213-220.

(4) Holt, D.L., Babcock, S.G., Green, S.J. and Maiden, C.J., The Strain-Rate Dependence of the Flow Stress in Some Aluminum Alloys, Trans. ASM, Vol. 60 (1967), pp. 152-159.

(5) 'Ogawa, K., Mechanical Behaviou'r of Titanium Alloys at High Rates of Strain, Plasticity and Impact Mechanics, N.K. Gupta, Editor, (1993), pp. 154-172, Wiley Eastern, New Delhi.

(6) Ogawa, K., Mechanical Behaviors of Beta-Titanium Alloy at High Rates of Strain, J. Phys. IV, France, Vol. 7 (1997), pp. 599-604.

(7) Kim, K.S. and Clifton, R.J., Pressure-Shear Impact of 6061-T6 Aluminum, J. Appl. Mech., Trans. ASME, Vol. 47 (1980), pp. 11-16.

(8) Conrad, H., Thermally Activated Deformation of Metals, J. Metals, Vol. 16 (1964), pp. 582-588.

(9) Kocks, U.F., Argon, A.S. and Ashby, M.F., Thermodynamics and Kinetics of Slip, (1975), Pergamon Press.

(10) Ledbetter, H.M., Temperature Behaviour of Young's Moduli of Forty Engineering Alloys, Cryogenics; Vol. 22, (1982), pp. 653-656.

(11) Kelly, A. and Nicholson, R.B., Precipitation Hardening, Chalmers, B., Editor; (1963), Pergamon Press, Oxford.

(12) Tanaka, K. and Nojima, T., Flow Stresses of Al and Al Alloys, The 21st Jap. Cong. Mat. Res., (1978), pp. 1-5.

(13) Zerilli, F.J. and Armstrong, R.W., DislocationMechanics-Based Constitutive Relations for Material Dynamics Calculations, J. Appl. Phys., Vol.61, No. 5 (1987), pp. 1816-1825. 